\title{
Distribución potencial del coatí (Nasua narica) en el noreste de México: implicaciones para su conservación
}

\author{
Claudia R. Espinoza-García ${ }^{1}$, Jesús M. Martínez-Calderas ${ }^{2 *}$, \\ Jorge Palacio-Núñez y Anuar D. Hernández-SaintMartín²
}

Introduction: From different ecological approaches, the coati (Nasua narica) is an important procyonid. Its distribution extends from southern of the United States to Colombia. IUCN (2013) considered it as Least Concern, but in Mexico is not considered under any category of protection due to its wide distribution. Despite this, there is poor knowledge about their population status and current distribution, especially in the northeastern region of the country, where in the last 30 years there have been important changes in land use. The landscape variability that establishes the limit of physiographic subprovinces (PSP) has implications for the availability and quality of habitat for many species, and so this criterion was used. The objective of this study was to estimate the coati potential distribution in four physiographic subprovinces in Northeastern Mexico.

Methodology: This study was conducted in northeastern Mexico, on the PSP: Gran Sierra Plegada (GSP), Carso Huasteco $(\mathrm{CH})$; Llanuras y Lomeríos (LL) and Llanura Costera Tamaulipeca (LCT). From estimates the potential distribution was used historical and recent records and 26 predictive variables. The records were obtained from online base dates and articles, and field wok from 2006-2012. This information was analyzed with Maxent algorithm version 3.3.3k (Phillips 2013), obtaining binary maps (presence-absence) using ArcMap 9.3 (ESRI 2006). The potential distribution, as a percentage of the total area, was calculated.

Results: We used 110 historical and recent records. Of these, 39 were obtained from databases and 71 fieldwork. The receiver operating characteristic (ROC) analysis showed a value of area under the curve (AUC) of $0.966 \pm 0.005$. Precipitation, vegetation type, vegetation cover, altitude, slope and temperature were the most relevant variables in explaining the potential distribution model we obtained, with contribution of $70 \%$ (Table 2). The potential distribution of coati covers $19.56 \%$ of the study area. The greatest distribution areas were found in the PSP's: GSP and CH, followed by LL and LCT.

Discussion and conclusions: Coati records for northeastern Mexico were found within a wide range of environmental conditions. Historical records obtained from databases are useful for the modeling of potential distribution, but it is essential to include current records (Pliscoff and Fuentes-Castillo 2011). According to ROC analysis, the model provided a good prediction. The environmental variables that explained the potential of coati distribution are similar to those mentioned in various studies as it is a species adapted to a wide range of altitude, temperature and precipitation. The PSP, GSP and CH met continuity of potential distribution. Despite this, the feasible area for the potential distribution of this species is greatly reduced, and the landscape in general was very fragmented. This fragmentation is a risk for long term viability of coati populations in Northeastern Mexico. It is proposed to establish management plans that combine agricultural production with elements that allow the distribution of this and other species, such as the planting of economic forests, as fruit orchards or timber.

\footnotetext{
'Instituto Tecnológico Superior de Irapuato. Carretera Irapuato - Silao, km. 12.5, Irapuato 36821. Guanajuato, México. E-mail: rocio.es25@gmail.com (CRE-G).

${ }^{2}$ Colegio de Postgraduados, Campus San Luis Potosí. Iturbide 73, Salinas de Hidalgo 78622, San Luis Potosí, México. E-mail: biologo99mx@yahoo.com.mx (JMM-C), jpalacio@colpos.mx (JP-N), anuarhernandez@gmail.com (ADH-S).

*Corresponding author
} 
Key words: Adaptability, connectivity, habitat fragmentation, habitat patches, land use changes, mediumsized mammals.

Resumen

El coatí (Nasua narica) es un prociónido importante desde diferentes enfoques ecológicos. El objetivo del presente estudio fue estimar su distribución potencial en cuatro subprovincias fisiográficas (SPF) en el noreste de México. Se usaron 110 registros entre históricos y recientes, y el algoritmo Maxent con 26 variables predictivas. La precipitación, tipo y cobertura vegetal, altitud, pendiente y temperatura fueron las variables más relevantes para explicar el modelo de distribución potencial resultante. La mayor distribución potencial se encontró en las SPF Gran Sierra Plegada y Carso Huasteco, seguidas por Llanuras y Lomeríos y, finalmente por la Llanura Costera Tamaulipeca. En las dos primeras SPF se encontraron zonas con continuidad de distribución potencial, pero el paisaje en general se encontró muy fragmentado, lo cual pone en riesgo la viabilidad de la especie en la zona estudiada a largo plazo.

Palabras clave: Adaptabilidad, cambios de uso del suelo, conectividad, fragmentación del hábitat, mamíferos medianos, parches de hábitat.

Introducción

El coatí o tejón (Nasua narica) es una de las siete especies de prociónidos presentes en México (Ramírez-Pulido et al. 2005; Ceballos y Arroyo-Cabrales 2012). Es un mamífero de talla mediana, de hábitos gregarios con manadas compuestas por hembras adultas y sus crías, mientras los machos adultos son solitarios (Valenzuela 2005). Su alimentación es omnívora; consume principalmente frutas e insectos, y en menor cantidad vertebrados pequeños (Gompper 1995; Valenzuela 1998). Es un importante dispersor de semillas (Russell 1982; Sáenz 1994) y forma parte de la dieta de los grandes depredadores tropicales como el puma y el jaguar (Núñez et al. 2000; Oliveira 2002; Weckel et al. 2006; Hernández-SaintMartín en prensa).

La distribución de N. narica se localiza en la región Neártica y Neotropical, desde el sur de Estados Unidos de América hasta Colombia (Gompper 1995; Samudio et al. 2008). Habita desde el nivel del mar hasta 3,500 m (Wilson y Reeder 2005) en hábitats boscosos templados y tropicales y, ocasionalmente en desiertos y sabanas (Gompper 1995). A nivel internacional, está considerado por la IUCN (2013) como preocupación menor (least concern). En México no se le considera bajo ninguna categoría de protección, debido a su amplia distribución, que incluye todos los estados, con excepción de la península de Baja California y las porciones más secas de la Altiplanicie Mexicana (Godínez-Navarro et al. 2008). Existe poca información sobre su estado poblacional, sobre todo para la porción noreste de México. Samudio et al. (2008) informan que probablemente sus poblaciones han decrecido o se han extirpado en ciertas regiones, debido principalmente a la pérdida y fragmentación de su hábitat, a la cacería ilegal y a las campañas de control de depredadores (e. g. Kaufmann et al. 1976).

En la actualidad, es común el uso de algoritmos para modelar la distribución potencial de las especies, que se basan en observaciones de campo y una serie de variables que actúan como predictores. El programa informático Maxent es un algoritmo robusto que 
modela la distribución potencial de especies por medio de la relación entre los puntos de presencia conocidos y las variables ambientales incluidas en el modelo (Phillips et al. 2006). A partir de esta relación, el algoritmo extrapola la presencia de la especie a las áreas donde no se cuenta con registros (Lindenmayer et al. 1991). El programa además estima la importancia de cada variable en la distribución de la especie y el modelo generado se valida con el área bajo la curva (AUC), derivada de la curva operada por el receptor (ROC), las cuales están implementadas en el software (Phillips et al. 2006).

El noreste de México ha tenido cambios en el uso de suelo, en particular en los últimos 30 años (Reyes-Hernández et al. 2007), por lo que la distribución actual de especies como el coatí en esta región no es muy bien conocida lo que complica la creación de planes efectivos de conservación. Solo existe un estudio sobre la distribución potencial coatí para México (Ceballos et al. 2006). Dicho estudio se realizó a una escala a nivel de país, y muestra a toda la región noreste como de mayor posibilidad de encontrar las condiciones favorables para la ocurrencia de esta especie. Es importante realizar estudios a una escala regional, incluyendo datos de presencia actuales. El presente trabajo se realizó con el objetivo de estimar la distribución potencial de esta especie en el noreste de México, mediante el modelado con base en registros históricos y recientes, que a su vez puedan servir de base para futuros planes de manejo en esta región.

Descripción del área de estudio. Este estudio se realizó en el noreste de México; que comprende el sur de Tamaulipas, el este de San Luis Potosí, el noreste de Querétaro, el noreste de Guanajuato, y en la porción norte de los estados de Hidalgo, Veracruz y Puebla (Fig. 1). La topografía de esta área va de muy escabrosa a ondulada y plana, con una serie de condiciones climáticas tropicales húmedas y sub-húmedas. La altitud varía de 0 a 2,300 msnm y la precipitación anual varia de 600 a 2,500 mm (INEGI 2011).

Las condiciones fisiográficas en el área de estudio son muy heterogéneas. Para definir mejor esta diversidad paisajística, se usó el criterio de provincias y subprovincias fisiográficas (SPF), las cuales se delimitan en función de su fluctuación topográfica y de la relación que esto tiene con el clima y con los tipos de vegetación (CervantesZamora et al. 1990). Con esta base, se incluyeron parcialmente a las provincias Sierra Madre Oriental y Llanura Costera del Golfo Norte, con la extensión total o parcial de dos subprovincias fisiográficas en cada una. La primera incluyó las SPF: Gran Sierra Plegada (GSP; 14,468 km²) y Carso Huasteco $\left(\mathrm{CH} ; 21,731 \mathrm{~km}^{2}\right)$; mientras que la segunda consideró parcialmente a Llanuras y Lomeríos (LL; 45,231 km²) y Llanura Costera de Tamaulipeca (LCT; 1,588 km²). El área total considerada es de 83,016 km². En la Figura 1 se muestran los límites estatales, así como la delimitación de las cuatro SPF consideradas en el presente estudio, y los sitios donde se ubican los registros del coatí, tanto históricos como recientes, que serán descritos más adelante.

Los tipos de vegetación en el área, determinada de la información de INEGI (2005) son: 1) antrópica, presente en áreas agrícolas o de vegetación inducida; 2) seca, compuesta por pastizal, matorral desértico, mezquital o vegetación halófita; 3) templada, compuesta por bosques, donde aparece el mesófilo de montaña, de pino, de encino, y sus asociaciones; 4) tropical seca, que incluye a los matorrales submontano y espinoso tamaulipeco, así como a las selvas bajas; 5) tropical húmeda, que comprende a las 
selvas medianas y altas y, 6) otros tipos de vegetación, donde se incluyeron: palmar, tular, popal y bosque de galería. En la Tabla 1 se muestran los seis porcentajes de los tipos de vegetación presentes en cada porción de las SPF consideradas en este estudio. En la GSP el uso del suelo es predominantemente antrópico donde domina la vegetación inducida para ganadería y la agrícola, le siguen la vegetación nativa tropical seca y la templada. En $\mathrm{CH}$ predominan el uso antrópico y la vegetación seca, seguidos por la vegetación tropical seca. En LL el uso antrópico es dominante, seguido por la vegetación tropical seca y la templada. Por último, la porción de LCT presenta un dominio amplio de vegetación de uso antrópico seguido de vegetación tropical seca y vegetación templada.

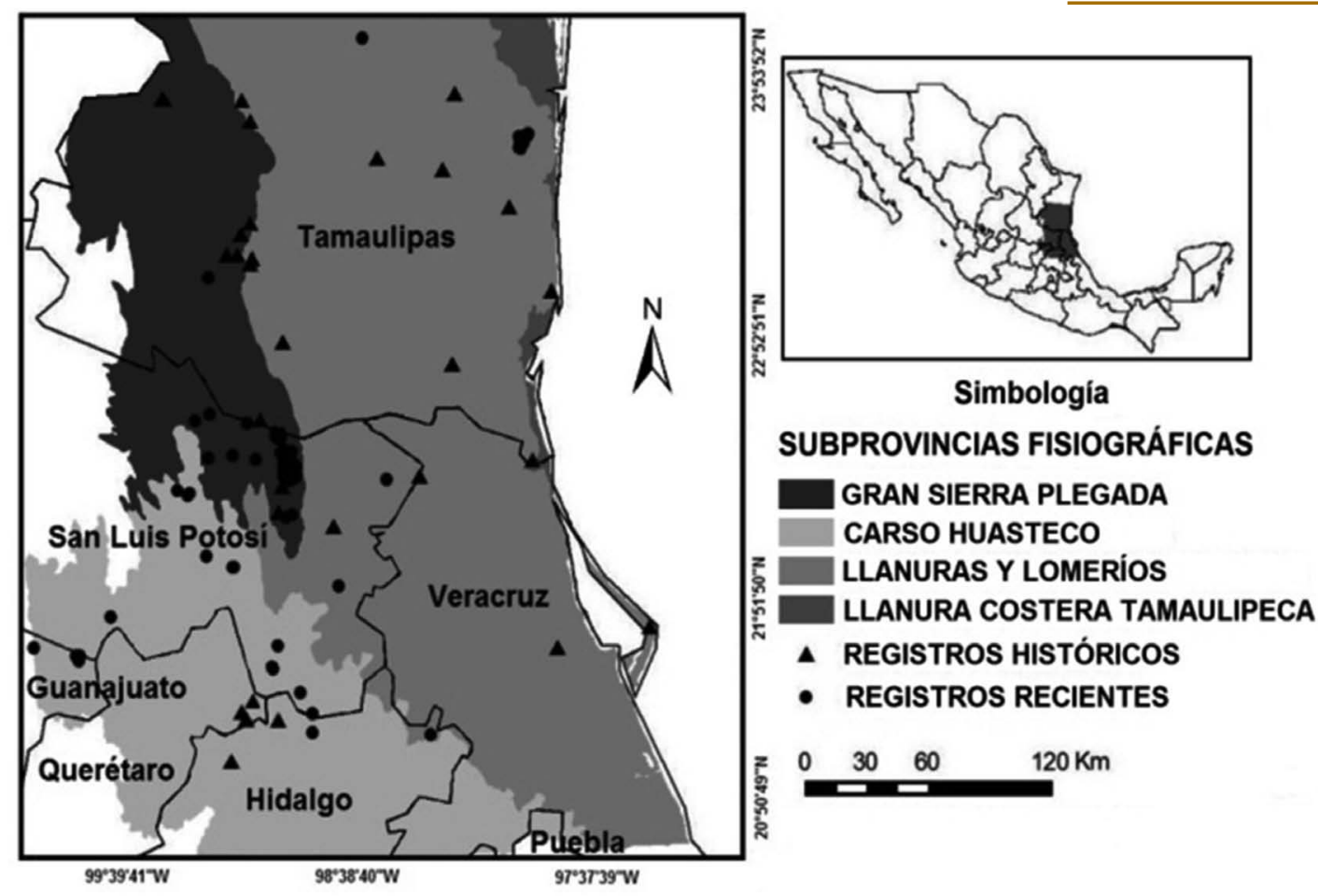

Figura 1. Mapa del área de estudio donde se muestra los estados y la localización geográfica de las subprovincias fisiográficas en el noreste de México, así como los puntos de registro del coatí, Nasua narica.

Obtención de registros y modelado. Se recabaron registros históricos y actuales del coatí, considerando a los primeros a los anteriores al año 2000 y los segundos a los posteriores de ese año. Esto debido a que la información disponible en la literatura y en bases de datos a partir de 2001 fue más afín a la de los datos propios, con registros obtenidos mediante el uso de trampas cámara. Los históricos se obtuvieron por medio del facilitador de bases de datos de Global Biodiversity Information Facility (GBIF; www.gbif.org), de la Unidad de Informática para la Biodiversidad (UNIBIO, www. unibio.unam.mx), de las bases de datos en línea de proyectos del Sistema Nacional de Información sobre Biodiversidad de la Comisión Nacional para el Conocimiento y uso de la Biodiversidad (SNIB-CONABIO; Fernández et al. 1998; López-Wilchis 1998; Ceballos 2002). Los registros recientes se obtuvieron mediante artículos científicos (Vargas-Contreras y Hernández-Huerta 2001), base de datos en línea de un proyecto de SNIB-CONABIO (Ceballos 2002) y datos propios derivados de trabajo de campo entre 2006-2012. 
Este trabajo de campo se realizó por medio de entrevistas, rastros y fototrampeo. Se realizaron 280 entrevistas en comunidades entre mayo de 2006 y agosto de 2010, siguiendo la técnica de Rabinowitz (1997). Fueron dirigidas a personas con experiencia de campo y capacidad para identificar correctamente las características distintivas e infalibles de la especie. Esto se enfocó en personas tales como peleteros, cazadores, veterinarios y ganaderos (tanto pequeños propietarios como ejidatarios).

Para analizar este tipo de información existe la técnica de Frey et al. (2013), que se basa en comparar la confiabilidad y la precisión de las respuestas. Sin embargo, en este trabajo se usó una clasificación según los criterios propuestos por Tewes y Everett (1986) dependiendo de la confiabilidad de las respuestas. En la clase I se consideraron aquellos con evidencia físicas, tales como pieles y cráneos en posesión de los entrevistados. Por su parte, la clase II consistió sólo en información detallada y creíble sobre el avistamiento de los coatíes, basándonos en una descripción confiable y en el posterior reconocimiento de la especie por parte del entrevistado, discerniendo entre varias fotografías de distintos animales (Rabinowitz 1997). Los registros de la clase III no fueron confiables y se desecharon.

Una vez obtenida la información y con el apoyo de guías locales, en su mayoría los mismos informantes, se procedió a visitar los sitios específicos de caza y/o avistamiento mencionados, los cuales se georreferenciaron con un geoposicionador (GPS, Garmin@ modelo GPS V). Con esto se obtuvieron datos adicionales sobre el lugar de cada registro, y se establecieron los sitios para el fototrampeo del primer periodo, que se realizó entre 2007 a 2008. El segundo periodo se llevó a cabo entre 2010 y 2012 en otra porción del área de estudio, y no incluyó entrevistas previas. En el primer periodo se usaron 20 trampas cámara de diferentes modelos, dejando hasta tres cámaras por localidad, con un esfuerzo de muestreo de 4 meses, dos en 2007 y dos en 2008 en 45 localidades, dando un total de 6,380 noches/trampa. Para el segundo periodo se instalaron 101 estaciones de fototrampeo dobles y sencillas, con un esfuerzo de cuatro meses cada año, dando un total de 7,583 noches/trampa.

Para estimar la distribución potencial del coatí dentro del área de estudio se empleó el algoritmo Maxent versión 3.3.3k (Phillips 2013), usando 26 variables ambientales como predictores. De éstas, 19 fueron variables bioclimáticas de México (Téllez et al. 2011). Otras cuatro fueron: pendiente, altitud, índice topográfico y aspecto topográfico, derivadas del modelo digital de elevación (INEGI 2008). También se incluyeron las variables tipo de vegetación con las seis categorías antes mencionadas (tomadas y modificadas de INEGI 2007) y la cobertura de la misma (tomadas de Hansen et al. 2000), y los tipos de suelo reportados por INEGI (2007). Todas las capas fueron usadas a una resolución de $1 \mathrm{~km}^{2}$ (0.01 pixeles).

Para generar el modelo general se obtuvo el promedio de 20 réplicas aleatorias, para lo cual se usó el 70\% de los datos de ocurrencia, mientras que el 30\% restante se usó para evaluar los errores de omisión y comisión, asignándolos al azar. Para esto se realizó una validación cruzada, lo que significa que los registros de presencia se pueden utilizar más de una vez en el conjunto de datos de validación para cada réplica, según especificaciones de Phillips et al. (2006) y Phillips (2013). Para la evaluación de cada modelo se consideró el valor del área bajo la curva (AUC) generada por el algoritmo (Hernadez et al. 2006). Esta fue obtenida directamente de la evaluación de los modelos 
por medio de las curvas ROC (e. g. Contreras-Medina et al. 2010), donde los valores de AUC cercanos a 1 demuestran que se trata de una buena predicción del modelo (Araujo y Guisan 2006).

\begin{tabular}{lllll}
\hline Subprovincia fisiográfica & & & & \\
\hline Tipo de vegetación & CH & GSP & LL & LCT \\
\hline Vegetación antrópica & 32.52 & 46.18 & 50.87 & 55.94 \\
Vegetación seca & 32.52 & 2.86 & 0.47 & 0.48 \\
Vegetación templada & 14.58 & 20.98 & 18.08 & 15.32 \\
Vegetación tropical seca & 18.75 & 27.00 & 25.27 & 24.95 \\
Vegetación tropical húmeda & 0.71 & 1.02 & 3.16 & 0.00 \\
Otro & 0.92 & 1.96 & 2.15 & 3.31 \\
\hline
\end{tabular}

Tabla 1. Porcentajes de los tipos de vegetación presentes en la porción de cada SPF incluida en el área de estudio.

Mediante una prueba de Jacknife se evaluaron las variables en el modelo y se obtuvo la información y porcentaje que aporta cada una, considerando que el mapa promedio representa el hábitat inferido y ajustado de la especie (Anderson et al. 2003; Burneo et al. 2009). El mapa obtenido se reclasificó con el sistema de información geográfica ArcMap 9.3 (ESRI 2006), obteniendo un mapa binario (presencia-ausencia), donde se aplicó un umbral de corte del mínimo de presencia de los puntos de entrenamiento ("mínimum training presence"; Pearson et al. 2007). Con este mapa y estos niveles se calculó el área de distribución potencial, expresado en porcentaje del área total de cada SPF.

Se obtuvo un total de 110 registros de presencia de coatí para el noreste de México en 108 localidades, con distancia variable entre ellas (de 2 a aproximadamente $80 \mathrm{~km}$ ), de los cuales 30 se adjudicaron a históricos y 80 a recientes. Sobre la procedencia de los registros, 21 resultaron de bases de datos de CONABIO, 11 de GBIF, 6 de UNIBIO, 1 de un artículo publicado y, finalmente, 71 resultaron del trabajo propio realizado en el área de estudio. Los registros históricos fueron: 10 avistamientos, 16 especímenes preservados en colecciones científicas y cuatro en los que no especifican el tipo de evidencia. Una observación final fue que, al analizar los puntos en la cartografía, se observó que el $53.33 \%$ de los registros históricos actualmente estaban localizados en áreas modificadas por actividades humanas, lo que sugiere cambios en el uso del suelo posteriores al año 2001; el resto se ubicaron en vegetación tropical seca (10.83\%), tropical húmeda (19.17\%) y vegetación templada (16.67\%).

En cuanto a los registros recientes, 7 se obtuvieron de base de datos: de estos, 2 fueron avistamientos y 5 obtenidos por fototrampeo. El único registro obtenido por artículo (Vargas-Contreras y Hernández-Huerta 2001) no especifica qué tipo de evidencia obtuvo. Los registros propios fueron 49 por fototrampeo, 19 por huella, 6 por avistamiento, 2 animales muertos en campo, 2 por entrevista y 2 por evidencia física en propiedad de los entrevistados (1 piel y 1 cráneo). Estos registros se localizaron en vegetación tropical húmeda $(45.86 \%)$ y tropical seca $(27.89 \%)$, seguido de antrópica $(16.25 \%)$ y de vegetación templada $(10 \%)$. No se apreciaron sesgos en la cantidad de registros en relación a las zonas con mayor número de pobladores humanos. 
De acuerdo con el análisis de la curva ROC, el modelo presentó una buena predicción $(A \cup C=0.966 \pm 0.005)$. Cinco variables de precipitación, cuatro de temperatura, dos de vegetación y dos derivadas del modelo digital de elevación, fueron las que contribuyeron con el $70 \%$ para la generación de este modelo promedio (Tabla 2). Referente a la distribución potencial del coatí, abarcó 16,238 km² (19.56\%) del área de estudio.

Respecto al porcentaje total para las SPF, en GSP hubo una probabilidad de presencia en $39.38 \%$ de su extensión, para $\mathrm{CH}$ fue $28.18 \%$, para LL fue 17.76 y $14.68 \%$ para LCT. En el noreste de México existen zonas con las condiciones apropiadas para la distribución de la especie, pero esas porciones están aisladas en parches de mayor o menor tamaño (Fig. 2). Las tres reservas de la biosfera ubicadas en esta región (El Cielo, Tamaulipas, Sierra del Abra-Tanchipa, San Luis Potosí y Sierra Gorda, Querétaro) presentaron áreas de distribución potencial.

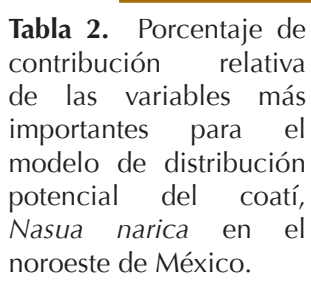

Tabla 2. Porcentaje de de las variables mán importantes para el modelo de distribución Nasua narica en el noroeste de México.

\begin{tabular}{ll}
\hline Variable & Contribución (\%) \\
\hline Precipitación del cuatrimestre más caliente & 17.2 \\
Tipo de vegetación & 12.3 \\
Precipitación estacional & 8.2 \\
Cobertura vegetal & 6.6 \\
Temperatura promedio del cuatrimestre más frío & 4.2 \\
Altitud & 4.0 \\
Pendiente & 3.8 \\
Precipitación del mes más caliente & 2.9 \\
Temperatura mínima del mes más frío & 2.8 \\
Precipitación del cuatrimestre más húmedo & 2.6 \\
Precipitación del cuatrimestre más frío & 2.2 \\
Temperatura del cuatrimestre más seco & 1.9 \\
Temperatura promedio anual & 1.4 \\
\hline
\end{tabular}

\section{Discusión}

La recopilación de registros depositados en diversas bases de datos es útil para la realización de modelos de distribución potencial. Sin embargo, los cambios en el uso del suelo en las localidades incluidas pueden generar sesgos, por lo cual es fundamental incluir registros actuales (Pliscoff y Fuentes-Castillo 2011; Frey et al. 2013), que permitan modelos más robustos y con menos errores. De hecho, en este trabajo, más de la mitad de registros históricos se localizaron en sitios donde hubo cambios en el uso del suelo (INEGI 2005) posteriores a la fecha de los registros. Este detalle fue observado ya al final del proceso, y el posible sesgo de este $14.5 \%$ de los datos no fue corregido. Este tipo de sesgo está presente en otros trabajos basados en un porcentaje mucho mayor de registros históricos. Así, en comparación con el mapa de distribución potencial para el coatí realizado por Ceballos et al. (2006), con base en 272 registros para todo México (sin especificar la procedencia), los resultados son totalmente diferentes. En dicho estudio se muestra que en el noreste del país existe el máximo valor de probabilidad de presencia 
de la especie, lo cual no ocurre con los resultados de este estudio. Es posible que la principal diferencia sea la escala del análisis y la cantidad de registros con los que se generó el modelo. Se ha demostrado que el uso de pocos registros en Maxent ocasionan una sobre-predicción en los modelos generados (Pearson et al. 2007). Por lo tanto, al aumentar el número de registros se puede mejorar la eficacia del modelo (Frey et al. 2013). En el presente estudio hubo una mayor densidad de registros para el noreste de México que el usado por Ceballos et al. (2006) en la modelación de todo el país.

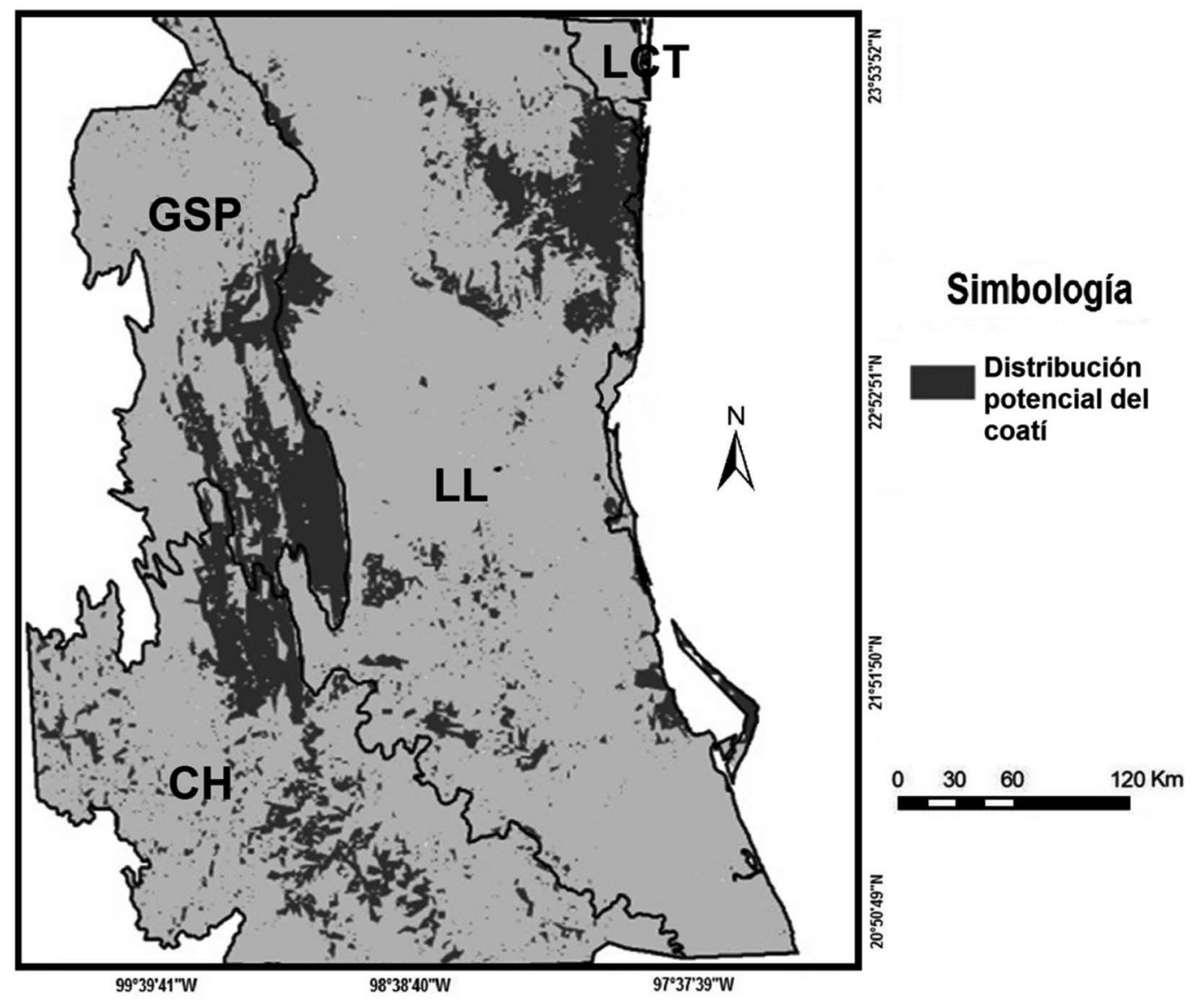

Figura 2. Modelo de distribución potencial del coatí, Nasua narica en el noreste de México, donde el color gris obscuro muestra los sitios con alta probabilidad de presencia. El gris claro muestra las porciones donde la distribución potencial no fue detectada, o no la hay. Abreviaturas: GSP = Gran Sierra Plegada, $\mathrm{CH}=$ Carso Huasteco, LL = Llanuras y Lomeríos, $\mathrm{LCT}=$ Llanura Costera Tamaulipeca.

El coatí es una especie presente en gran diversidad de hábitat, y el área de estudio de este trabajo también tuvo una gama de variantes paisajísticas que propician diferentes ambientes. En relación a su distribución potencial, la mayor proporción se localizó en las SPF GSP (39.4\%) y CH (28.2\%), que presentaron las mayores porciones de sitios de cobertura arbórea densa, de acuerdo con lo reportado por Sahagún-Sánchez et al. (2011) para esta misma región. En estas dos subprovincias se observó la mayor extensión continua de distribución potencial. Sin embargo, el paisaje fragmentado por vegetación antrópica (áreas agrícolas o vegetación inducida) ocupa una porción importante en ambas. Las reservas de la biosfera El Cielo, y Sierra del Abra-Tanchipa se encuentran en GSP, y ambas quedaron plenamente en el área de distribución potencial de esta especie, compuesta principalmente por vegetación templada, seca y tropical seca. En el caso de Sierra Gorda, está en $\mathrm{CH}$ y la distribución potencial del coatí se encontró sólo en parches. En el caso de LL, la vegetación antrópica tuvo un porcentaje mayor (50.9\%); sin embargo, existe una distribución potencial continua del coatí en un área 
considerable al noreste de esta SPF en áreas con vegetación nativa; en el resto de la extensión sólo se observaron parches. En la LCT. Fue donde se presentó la menor área de distribución, ya que es la que presenta menor proporción de hábitat apropiado, tal como vegetación templada y tropical seca y húmeda (Martínez-Calderas 2009).

En el modelo, 13 (de 26) variables ambientales explicaron la distribución potencial del coatí en el noreste de México y fueron las relacionadas con la precipitación, el tipo y cobertura de la vegetación, la altitud y la pendiente del terreno, así como la temperatura. Respecto a la influencia de las variables en la distribución potencial, para la precipitación y la temperatura no es de esperar cambios sensibles de un lado a otro en las fronteras de las SPF; sin embargo, el tipo de vegetación y la cobertura de la misma, la altitud y la pendiente del terreno sí pueden cambiar.

El coatí es una especie presente en gran diversidad de hábitats, y para el noreste de México, se encontró dentro de una amplia gama de condiciones ambientales, presente principalmente en áreas tropicales y subtropicales, también está adaptada a la gama de altitud, temperatura y precipitación presente en estos ecosistemas (Scholander et al. 1950; Gompper 1995; Valenzuela 1998; Valenzuela 2005; Samudio et al. 2008). Al igual que muchas otras especies de mamíferos, presenta una relación entre su periodo reproductivo con la oscilación de la precipitación y la disponibilidad de alimento (Gompper 1997; Valenzuela 1998; Valenzuela y Macdonald 2002), independientemente si el hábitat es más o menos húmedo. Su ámbito hogareño fluctúa a través del año, siendo mayor en época seca, cuando es necesaria una mayor movilidad por mayor dispersión de los recursos (Sáenz 1994; Gompper 1997; Valenzuela y Ceballos 2000; Hass 2002).

El coatí habita preferentemente en sitios arbolados, desde bosques templados a selvas tropicales húmedas y vegetación tropical seca (Ceballos y Galindo 1984; Ratnayeke et al. 1994; Gompper 1995; Valenzuela 1998; Valenzuela 2005; Samudio et al. 2008), aunque también se le encuentra eventualmente en vegetación seca, entre la que figuran desiertos y pastizales (Gompper 1995; Samudio et al. 2008). Su presencia en pastizales se ha reportado muy poco (Sáenz 1994), pero cuando la cobertura de los pastos es alta, este ecosistema le brinda protección contra depredadores (Endres y Smith 1993). En relación a la importancia de las variables topográficas (altitud y pendiente) en el modelo de distribución esta especie no existen referencias sobre el tema. En general, los sitios con mayor pendiente favorecen la protección contra depredadores para varias especies de mamíferos, y son áreas menos propensas al impacto humano directo y, por lo tanto, suelen conservar hábitat menos perturbado (Badgley y Fox 2000; Campbell 2004).

Actualmente en la zona de estudio el paisaje está muy fragmentado, existen remanentes de vegetación cerrada básicamente en terrenos con pendientes pronunciadas, frecuentemente escabrosas, lo que es impráctico para la agricultura y la ganadería (Guevara y Laborde 1999; Trejo y Dirzo 2000; Reyes-Hernández et al. 2007). La fragmentación del hábitat altera la composición y estructura de las comunidades animales por modificación de varios procesos ecológicos (Wilcove 1985). La supervivencia de las especies en los hábitat fragmentados finalmente depende de su habilidad para moverse entre parches y acceder a los recursos necesarios, conservar su diversidad genética y mantener su capacidad reproductiva como población (Laurance 
1995; Wolff 1999; Buza et al. 2000; Nupp y Swihart 2000; Gehring y Swihart 2004), así como por la disposición de los elementos espaciales (Gehring 2000).

Algunos estudios sugieren que incluso animales extremadamente móviles pueden evitar pasar a través de hábitat alterados (Smallwood 1994; Machtans et al. 1996). Por esto, si hay interrupción en la conectividad entre dos o más pequeñas poblaciones, se puede llegar a una extinción local (Beier 1993). Esto no es una excepción para el coatí, cuyas poblaciones se han reducido en algunos lugares desde hace décadas por la pérdida de su hábitat (Glatston 1994; Gompper 1995; Samudio et al. 2008). Por esto, un paisaje fragmentado no garantiza su viabilidad a mediano y largo plazo. Por lo tanto, las zonas contiguas entre la porción sur de GSP, con un fragmento al oeste de LL, y con el norte de $\mathrm{CH}$, así como la zona noreste de LL (ver Fig. 2) se proponen como las más importantes por mantener una distribución continua, que de este modo puede permitir la conservación de Nasua narica en el noreste de México. En general, para mitigar efectos de fragmentación, la plantación de bosques útiles (frutales o maderables) más que la producción de cultivos anuales, con especies no necesariamente nativas, puede proporcionar hábitat adecuado para especies adaptables (Martin et al. 2012) como el coatí. Esta estrategia es acorde con tipos de uso de suelo antrópico en esta parte del país (Jiménez et al. 2004), y su incremento puede beneficiar tanto a la economía local como a algunas especies de fauna silvestre, entre ellas al coatí.

Araujo, M. B., y A. Guisan. 2006. Five (or so) challenges for species distribution modelling. Journal of Biogeography 33:1677-1688.

Anderson, R. P., D. Lew, y A. T. Peterson. 2003. Evaluating predictive models of species distributions: criteria for selecting optimal models. Ecological Modelling 162:211232.

Badgley, C., y D. L. Fox. 2000. Ecological biogeography of North American mammals: species density and ecological structure in relation to environmental gradients. Journal of Biogeography 27:1437-1467.

Beier, P. 1993. Determining minimum habitat areas and habitat corridors for cougars. Conservation Biology 7:94-108.

Burneo, S., J. F. González-Maya, y D. Tirira. 2009. Distribution and habitat modelling for Colombian weasel Mustela felipei in the Northern Andes. Small Carnivore Conservation 41:41-45.

Buza, L., A. Young, y P. Thrall. 2000. Genetic erosion, inbreeding and reduced fitness in fragmented populations of the endangered tetraploid sea Swaisona recta. Biological Conservation 93:177-186.

Campbell, L. A. 2004. Distribution and habitat associations of mammalian carnivores in the Central and Southern Sierra Nevada. Tesis de Doctorado, University of California. Sacramento, EE.UU.

Ceballos, G., y C. Galindo. 1984. Mamíferos Silvestres de la Cuenca de México. LIMUSA-Instituto de Ecología. Ciudad de México, México.

Ceballos, G. 2002. Actualización de la base de datos del Atlas Mastozoológico de México. Universidad Nacional Autónoma de México. Instituto de Ecología. Bases de datos SNIB2010-CONABIO proyectos No. T009 y A003. Ciudad de México, México. 
Ceballos, G., S. Blanco, C. González, y E. Martínez. 2006. Nasua narica (Tejón, coatí). Distribución potencial. Extraído del proyecto DS006 'Modelado de la distribución de las especies de mamíferos de México para un análisis GAP'. Con un tamaño de píxel: 0.01 grados decimales. Instituto de Biología, Universidad Nacional Autónoma de México (UNAM). Financiado por la Comisión Nacional para el Conocimiento y Uso de la Biodiversidad (CONABIO). Ciudad de México, México.

Ceballos, G., y J. Arrollo- Cabrales. 2012. Lista actualizada de los mamíferos de México 2012. Revista Mexicana de Mastozoología Nueva época 2:27-80.

Cervantes-Zamora, Y., S. L. Cornejo-Olgín, R. Lucero-Márquez, J. M. Espinoza-Rodríguez, E. Miranda-Viquez, y A. Pineda-Velázquez. 1990. Provincias Fisiográficas de México. Clasificación de Regiones Naturales de México II, IV. Versión 10.2. Atlas Nacional de México. Vol. II. Escala 1:4,000,000. Instituto de Geografía, Universidad Nacional Autónoma de México. Ciudad de México, México.

Contreras-Medina, R., I. Luna-Vega, y C. A. Ríos-Muñoz. 2010. Distribución de Taxus globosa (Taxaceae) en México: Modelos ecológicos de nicho, efectos del cambio del uso de suelo y conservación. Revista Chilena de Historia Natural 83:421-433.

Endres, K., Y W. Sмith. 1993. Influence of age, sex, season and availability on den selection by raccoons within the central basin of Tennessee. American Midland Naturalist 129:116-131.

EsRı. 2006. ArcGIS Desktop 9.2. Environmental System Research Institute. Palm Springs, EE.UU.

Fernández, V., A. Hernández-Huerta, y J. Bello. 1998. Inventario de los mamíferos de las reservas de la biósfera Mapimí, La Michilía, El Cielo y Calakmul. Instituto de Ecología A.C. Bases de datos SNIB2010-CONABIO proyecto No. P027. Ciudad de México, México.

Grif Gobal Biodiversity Information Facility. www.gbif.org. Consultada el 25 de enero de 2013.

Gehring, T. M. 2000. Ecology of mammalian predators in a landscape fragmented by agriculture. Tesis de doctorado. Universidad Purdue. West Lafayette, EE.UU.

Gehring, T. M., y R. K.v SwiHart. 2004. Home range and movements of long-tailed weasels in a landscape fragmented by agriculture. Journal of Mammalogy 85:79_ 86.

Glatston, A. R. 1994. The Red Panda, Olingos, Coatis, Raccoons, and their relatives. Status Survey and Conservation Action Plan for Procyonids and Ailurids. IUCN/ SSC Mustelid, Viverrid and Procyonid Specialist Group. Gland, Suiza.

Godínez-Navarro, E., A. Río-Vélez, H. Covarrubias-Legaspi, y R. Velázquez-López. 2008. Guía de mamíferos de Arcediano. Gobierno del Estado de Jalisco. Guadalajara, México.

GompPer, M. 1995. Nasua narica. Mammalian Species 487:1-10.

Gomprer, M. 1997. Population ecology of the white-nosed coati (Nasua narica) on Barro Colorado Island, Panama. Journal of Zoology 241:441-455.

Guevara, S., y J. Laborde. 1999. Historia del paisaje de la sierra de Los Tuxtlas, Veracruz (México). Pp. 44-64 in Diversidad biológica y cultural rural: en la gestión ambiental de desarrollo (Pineda, F. D., J. M. de Miguel, y M. A. Casado, eds.). Mundiprensa y Multimedia Ambiental. Madrid, España. 
Hansen, M., R. Defries, J. R. G. Townshend, y R. Sohlberg. 2000. Global land cover classification at $1 \mathrm{~km}$ resolution using a decision tree classifier. International Journal of Remote Sensing 21:1331-1365.

Hass, C. 2002. Home-range dynamics of white-nosed coatis in Southeastern Arizona. Journal of Mammalogy 83:934-946.

Hernandez, P. A., C. H. Graham, L. L. Master y D. L. Albert. 2006. The effect of sample size and species characteristics on performance of different species distribution modeling methods. Ecography 29:773-785.

Hernández-SaintMartín, A. D., O. C. Rosas-Rosas, J. Palacio-Núñez, L. A. TarangoArámbula, F. Clemente-Sánchez, y A. L. Hoogesteijn. En prensa. Food habits of jaguar and puma in a protected area and adjacent fragmented landscape of Northeastern Mexico. Natural Areas Journal.

Inegi (Instituto Nacional de Estadística y Geografía). 2005. Conjunto de datos Vectoriales de la Carta de Uso del Suelo y Vegetación, Escala 1:250,000, Serie III, Continuo Nacional. Instituto Nacional de Estadística, Geografía e Informática (INEGI). Ciudad de México, México.

Inegi (Instituto Nacional de Estadística y Geografía). 2007. Conjunto de datos Vectoriales Edafológicos. Escala 1:250,000, Serie II, Continuo Nacional. Instituto Nacional de Estadística, Geografía e Informática (INEGI). Ciudad de México, México.

Inegi (Instituto Nacional de Estadística y Geografía). 2008. Continuo de Elevación. Nacional. Instituto Nacional de Geografía e Informática http://mapserver.inegi.org. mx. Consultada el 11 de junio de 2012. Aguascalientes, México.

Inegi (Instituto Nacional de Estadística y Geografía). 2011. Cartas geográficas de clima, suelo y precipitaciones, Escalas 1: 100,000 y 1: 250,000, Instituto Nacional de Estadística, Geografía e Informática. Ciudad de México, México.

IuCN (INTernational Union for Conservartion of Nature). 2013. IUCN Red List of Threatened Species. Versión 2013.2. www.iucnredlist.org. Consultada el 11 de marzo de 2013.

Jiménez, C. A., V. T. Vargas, W. E. Salinas, M. Aguirre, y D. Rodríguez. 2004. Aptitud agroecológica para el cultivo de la caña de azúcar en el sur de Tamaulipas, México. Investigaciones Geográficas, Boletín del Instituto de Geografía Universidad Nacional Autónoma de México 53:58-74.

Kaufmann, J. H., D. V. Lanning, y S. C. Poole. 1976. Current status and distribution of the coati in the United States. Journal of Mammalogy 57:621-637.

Laurance, W. F. 1995. Extinction and survival of rainforest mammals in a fragmented tropical landscape. Pp. 46-63 in Landscape Approaches in Mammalian Ecology and Conservation (Lidicker Jr., W. Z. eds.). University of Minnesota. Minneapolis, EE.UU.

Lindenmayer, D., H. Nix, J. Mcmahon, M. Hutchison, y M. Tanton. 1991. The conservation of leadbeater's possum, Gymnobelideus leadbeateri McCoy, a case study of the use of bioclimatic modelling. Journal of Biogeography 18:371-383.

López-WıLChIs, R. 1998. Base de datos de mamíferos de México depositados en colecciones de Estados Unidos y Canadá. Universidad Autónoma Metropolitana-Iztapalapa. Informe final SNIB-CONABIO proyecto No. P130. Ciudad de México, México. 
Machtans, C. S., M.A. Villard, y S. J. HanNon. 1996. Use of riparian buffers strips as movement corridors by forest birds. Conservation Biology 10:1377-1379.

Martin, P. S., C. Gheler-Costa, P. C. Lopes, L. M. Rosalino, y L. M. Verdade. 2012. Terrestrial non-volant small mammals in agro-silvicultural landscapes of Southeastern Brazil. Forest Ecology and Management 282:185-195.

Martínez-Calderas, J. 2009. Nuevos registros y distribución del ocelote (Leopardus pardalis) en el noreste de México, Tesis de Maestría. Colegio de Postgraduados. Montecillo, México.

Nupp, T. E., Y R. K. Swinart. 2000. Landscape-level correlates of small mammal assemblages in forest fragments of farmland. Journal of Mammalogy 81:512-526.

Núñez, R., B. Miller, y F. Lindzey. 2000. Food habits of jaguars and pumas in Jalisco, Mexico. Journal of Zoology 252:373-379.

Oliveira, T. 2002. Ecología comparativa de la alimentación del jaguar y el puma en el Neotrópico. Pp. 265-288 en El jaguar en el Nuevo Milenio (Medellín, R. A., C. Equihua, C. Chetkiewics, A. Rabinowitz, P. Crawshaw, K. Redford, J. Robinson, y A. Taber, eds.). Fondo de Cultura Económica-UNAM-Wildlife Conservation Society. Ciudad de México, México.

Pearson, R. G., C. J. Raxworthy, M. Nakamura, A. T. Peterson. 2007. Predicting species distributions from small numbers of occurrence records: a test case using cryptic geckos in Madagascar. Journal of Biogeography 34:102-117.

Philuips, S. J., R. P. Anderson, y R. E. Schapire. 2006. Maximum entropy modeling in species geographic distributions. Ecological Modeling 190:231-259.

Philusps, S. J. 2013. A brief tutorial on Maxent, Versions: 3.3.1. www.cs.princeton.edu / schapire/maxent/. Consultada el 19 de mayo de 2013.

Puiscoff, P., y T. Fuentes-Castillo. 2011. Modelación de la distribución de especies y ecosistemas en el tiempo y en el espacio: una revisión de las nuevas herramientas y enfoques disponibles. Revista de Geografía Norte Grande 48:61-79.

Ramírez-Pulido, J., J. Arroyo-Cabrales, y A. Castro-Campillo. 2005. Estado actual y relación nomenclatural de los mamíferos terrestres de México. Acta Zoológica Mexicana (n. s.) 21:21-82.

Ratnayeke, S., A. Bixler, y J. Gittleman. 1994. Home range movements of solitary, reproductive female coatis, Nasua narica, in south eastern Arizona. Journal of Zoology 233:322-326.

Rabinowitz, A. R. 1997. Wildlife field research and conservation training manual. Wildlife Conservation Society. New York, EE.UU.

Reyes-Hernández, H., M. Aguilar-Robledo, J. R. Aguirre-Ribera, M. Silva-Aparicio, y I. R. Trejo-VÁzQuez. 2007. Caracterización de remanentes arbóreos y razones de su permanencia en el área del proyecto Pujal-Coy, San Luis Potosí, México. Pp. 85104 en Corredores biológicos: acercamiento conceptual y experiencias en América (Chassot, O., y C. Morera, eds.). Centro Científico Tropical, Universidad Nacional de Costa Rica/Escuela de Ciencias Geográficas, Instituto Panamericano de Geografía e Historia. Sanjosé, Costa Rica. 
RusselL, J. K. 1982. Timing of reproduction by coatis (Nasua narica) in relation to fluctuations in food resources. Pp. 413-431 en The ecology of a tropical forest: seasonal rhythms and long term changes (Leihg, E., A. Rand, y D. Windsor, eds.). Smithsonian Institute. Washington, EE.UU.

SáENZ, J. 1994. Ecología del pizote (Nasua narica) y su papel como dispersador de semilla en el bosque seco tropical, Costa Rica. Tesis de Maestría, Universidad Nacional San José, Costa Rica. San José, Costa Rica.

Sahagún-Sánchez, F. J., H. Reyes-Hernández, J. L. Flores -Flores, y L. Chapa-Vargas. 2011. Modelización de escenarios de cambio potencial en la vegetación y el uso de suelo en la Sierra Madre Oriental de San Luis Potosí, México. Journal of Latin American Geography 10:65-86.

Samudio, R., R. Kays, A. D. Cuarón, J. L. Pino, y K. Helgen. 2008. Nasua narica. In UiCN 2013. IUCN Red List of Threatened Species, Versión 2013.1. www.iucnredlist.org. Consultada el 11 de marzo de 2013.

Scholander, P. F., R. Hock, V. Walters, y L. Irving. 1950. Adaptation to cold in artic an tropical mammals and birds in relation to body temperature, insulation, and basal metabolic rate. Biological Bulletin 99:259-271.

Smallwood, K. S. 1994. Trends in California mountain lion population. The Soutwestern Naturalist 39:67-72.

Téllez, O., A. Hutchinson, A. Nix, y P. Jones. 2011. Cambio Climático: Aproximaciones para el estudio de su efecto sobre la biodiversidad. Pp. 15-23 en Desarrollo de Coberturas Climáticas para México (Sánchez-Rojas, G., C. Ballesteros-Barrera, y N. Pavón, eds.). Universidad Autónoma de Hidalgo. Pachuca, México.

Tewes, M. E., y D. Everett. 1986. Status and distribution of the endangered ocelot and jaguarondi in Texas. Pp. 147-158 en Cats of the world: biology, conservation, and management (Miller, S. D., y D. D. Everett, eds.). National Wildlife Federation. Washington, EE.UU.

Trejo, I., Y R. Dirzo. 2000. Deforestation of seasonally dry tropical forest: a national and local analysis in Mexico. Biological Conservation 94:133-142.

UNıBıO. Unidad Informática para la Biodiversidad. www.unibio.unam.mx. Consultado el 25 de enero de 2013.

Valenzuela, D. 1998. Natural history of the white-nosed coati, Nasua narica, in a tropical dry forest of western Mexico. Revista Mexicana de Mastozoología 3:26-44.

Valenzuela, D. 2005. Tejón, Coatí. Nasua narica (Linnaeus, 1766). Pp. 411-413 en Los Mamíferos Silvestres de México (Ceballos, G., y G. Oliva, coords.). Fondo de Cultura Económica / CONABIO. Ciudad de México, México.

Valenzuela, D., y G. Ceballos. 2000. Habitat selection, home range, and activity of the white-nosed coati (Nasua narica) in a Mexican tropical dry forest. Journal of Mammalogy 81:810-819.

Valenzuela, D., y D. Macdonald. 2002. Home range use by white-nosed coatis: limited water and a test of the Resource Dispersion Hypothesis. Journal of Zoology 258:247-256.

Vargas-Contreras, J. A., y A. Hernández-Huerta. 2001. Distribución altitudinal de la mastofauna en la Reserva de la Biosfera El Cielo, Tamaulipas, México. Acta Zoológica Mexicana (n. s.) 82:83-109. 
Weckel, M., W. Giuliano, y S. Silver. 2006. Cockscomb revisited: jaguar diet in the Cockscomb Basin Wildlife Sanctuary, Belize. Biotropica 38:687-690.

Witcove, D. S. 1985. Nest depredation in forest tracts and the decline of migratory songbirds. Ecology 66:1211-1214.

Wilson, D. E., y D. M. Reeder (eds.). 2005. Mammals Species of the World, a Taxonomic and Geographic Reference, tercera edición. Johns Hopkins Press. Baltimore, EE.UU.

Wolff, J. O. 1999. Behavioral model systems. Pp. 11-40 en Landscape ecology of small mammals (Barrett, G.W., y J. D. Peles, eds.). Springer-Verlag. NewYork, EE.UU.

Sometido: 19 de febrero de 2014

Revisado: 31 de marzo de 2014

Aceptado: 11 de abril de 2014

Editor asociado: Consuelo Lorenzo

Diseño gráfico editorial: Gerardo Hernández 
